\title{
Microscopic origins of heliclinic phases in smectic liquid crystals
}

Jonathan J. Stott

Fairfield University, jstott@fairfield.edu

Rolfe G. Petschek

Follow this and additional works at: https://digitalcommons.fairfield.edu/physics-facultypubs

(C1999 American Physical Society

The final publisher PDF has been archived here with permission from the copyright holder.

\section{Peer Reviewed}

\section{Repository Citation}

Stott, Jonathan J. and Petschek, Rolfe G., "Microscopic origins of heliclinic phases in smectic liquid crystals" (1999). Physics Faculty Publications. 114.

https://digitalcommons.fairfield.edu/physics-facultypubs/114

\section{Published Citation}

Stott, Jonathan J., and Rolfe G. Petschek. "Microscopic origins of heliclinic phases in smectic liquid crystals." Physical Review E 60, no. 6 (1999): 6826.

This item has been accepted for inclusion in DigitalCommons@Fairfield by an authorized administrator of DigitalCommons@Fairfield. It is brought to you by DigitalCommons@Fairfield with permission from the rightsholder(s) and is protected by copyright and/or related rights. You are free to use this item in any way that is permitted by the copyright and related rights legislation that applies to your use. For other uses, you need to obtain permission from the rights-holder(s) directly, unless additional rights are indicated by a Creative Commons license in the record and/or on the work itself. For more information, please contact digitalcommons@fairfield.edu. 


\title{
Mî́cróscopic origins of heliclinic phasès 'in' smectic liquid crystals
}

\author{
$\therefore \quad$ Jonathan J. Stott and Rolfer. G. Petschek \\ Department of Physics, Case Western Reserve Uñiversity, Cleveland, Ohio 44106-7079 \\ (Received 21 June 1999 )
}

\begin{abstract}
In a previous article [Phys. Rev. E 60, 1799 (1999)], the authors considered a model Landau free energy that explained the ferriclinic phases of chiral smectic liquid crystals as a series of short period helical modulations. In this paper we begin with a physically more realistic, more microscopic interlayer free energy and show how our previous work can be derived using only simple short-ranged interactions. We then discuss what additional information this provides about the Landau coefficients used previously to construct the phase diagram for the heliclinic phases of chiral smectic liquid crystals. Finally, we investigate a means for explicitly including chirality in our model. [S1063-651X(99)02412-5]
\end{abstract}

PACS number(s): $64.70 . \mathrm{Md}, 61.30 . \mathrm{Cz}$

\section{INTRODUCTION}

In smectic liquid crystals, the molecules self-assemble into periodic layered structures. By convention, the layer normal defines the $z$ axis of the system. The molecules of the liquid crystal are anisotropic which often leads to pronounced birefringence effects. For the systems we will consider, the molecules can be viewed as elongated ellipsoids, in which case the long axis of the molecule coincides with the extraordinary index of refraction.

The orientation of the liquid crystal molecules is represented by a unit vector $\hat{\vec{n}}$ called the director. By convention, the director is along the optical axis of the molecule. In the smectic- $A$ (Sm- $A$ ) phase, the director is parallel to the layer normal. In the Sm-C phases, the director tilts and develops a component perpendicular to the layer normal. A tilt vector $\vec{c}$ can be used to represent this tilt. The tilt vector is.constrained to lay in the plane of the smectic layers and its magnitude is equal to the projection of the director onto the plane of the layers,

$$
|\vec{c}|=|\hat{\vec{z}} \times \hat{\vec{n}}| .
$$

In the nonchiral $\mathrm{Sm}-C$ phase, the molecules, on average, tilt in the same direction throughout the sample. In a chiral material, on the other hand, as one moves along the $z$ axis the tilt direction precesses about the layer normal with a period much larger than the layer spacing. This is known as the $\mathrm{Sm}-C^{*}$ phase. Because these molecules lack a center of inversion, the $\mathrm{Sm}-C^{*}$ phase usually has a spontaneous ferroelectric polarization [1]. This polarization couples strongly to an applied electric field which has applications in the manufacture of optical devices.

Ferroclinic phases (also known as synclinic phases) are liquid crystal phases where the average tilt vector points in the same direction from layer to layer (ignoring any rotations due to the chirality). In antiferroclinic (syndioclinic) phases, on the other hand, the tilt vector changes direction between adjacent layers $\vec{c}_{\ell}=-\vec{c}_{\ell+1}$. Ferriclinic phases are the intermediate case where the tilt vector is neither parallel nor antiparallel to the adjacent layers. In chiral materials, these phases also possess spontaneous polarizations; chiral ferroclinics are necessarily ferroelectric. In a previous paper [2], they are modeled for the ferriclinic smectic phases as a series of short period helical modulations about the layer normal in the context of a Landau free energy. Such a heliclinic phase is shown.in Fig. 1. The tilt in each of five successive layers is illustrated by drawing an arrow on a cone. The particular phase illustrated is a chiral phase with a period of four lattice spacings. A variety of other phases can exist, depending on the period with which the director repeats, and the nature of the modulation within this period. From this, under certain simplifying assumptions, we derived the phase diagram and characterized the various phases.

On doing so we found a number of ferrielectric phases. One bore a strong resemblance to the smectic- $C_{\gamma}$ phase. Another, which consisted of a helical modulation with a period of three times the layer spacing, may have been experimentally observed by Mach and collaborators [3]. In addition, our model predicts two other ferriclinc phases which do not seem to correspond to any phases yet observed. Finally, we demonstrated the importance of mode locking between different Fourier modes in determining the phase diagram.

While the Landau free energy formulation we used is convenient to work with, it is not always the most satisfying approach. In particular, the Landau free energy gives very few clues as to the sign or magnitude of the various phenomenological coefficients except what can be inferred from physical arguments. Being a phenomenological theory, no indication is provided as to the physical origin of the various terms either. Finally, in our previous investigations [2], while we implicitly assumed the existence of chirality, we did not treat it explicitly. In this paper we will attempt to remedy these shortcomings and to provide a more concrete framework for the heliclinic phases.

\section{AN INTERLAYER FREE ENERGY}

Our starting point for improving our understanding of the heliclinic phases is an interlayer free energy with interactions

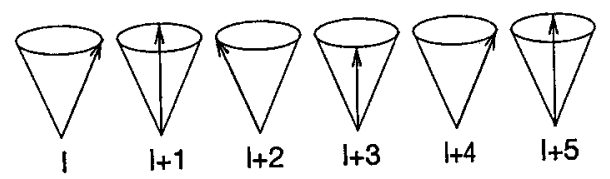

FIG. 1. Illustration of a heliclinic phase $(q=2 \pi / 4 a$ in this example). 
between pairs of molecular tilts in smectic layers

$$
\begin{aligned}
\mathcal{F}= & \frac{1}{2} \sum_{m, n}\left(J_{n, m}\left(\vec{c}_{n} \cdot \vec{c}_{m}\right)+J_{n, m}^{\prime}\left(\vec{c}_{n} \times \vec{c}_{m}\right) \cdot \hat{\vec{z}}\right) \\
& +\frac{1}{4} \sum_{m, n}\left(V_{n, m}\left(\vec{c}_{n} \cdot \vec{c}_{m}\right)^{2}\right. \\
& \left.+V_{n, m}^{\prime}\left(\vec{c}_{n} \cdot \vec{c}_{n}\right)\left(\vec{c}_{m} \cdot \vec{c}_{m}\right)\right)+O\left(c^{6}\right) .
\end{aligned}
$$

Here, $J_{n, m}$ contains the nonchiral interaction (e.g., steric forces, van der Waals, elastic, dipole-dipole, etc. [4]) between molecules and $J_{n, m}^{\prime}$ contains the chiral interactions. The tilt vector for a given molecule $n$ is given by $\vec{c}_{n} . V_{n, m}$ and $V_{n, m}^{\prime}$ are the fourth-order interaction terms. We assume that any chiral fourth-order terms are small enough that they may be safely neglected. We also assume that the long-range fourth-order terms $\left(\vec{c}_{k} \cdot \vec{c}_{l}\right)\left(\vec{c}_{n} \cdot \vec{c}_{m}\right)$ with $k \neq l \neq n$ may also be safely.neglected. Finally we assume, as is commonly done when modeling phase transitions, that the second-order terms vary with temperature while the fourth-order terms are temperature independent.

Based on the symmetry of real systems, all the coupling terms must be translationally invariant. Further, only terms invariant under the interchange $n \leftrightarrow m$ can contribute to the free energy, from which it immediately follows that

$$
\begin{gathered}
J_{n, m}=J_{m, n}, \\
J_{n, m}^{\prime}=-J_{m, n}^{\prime}, \\
V_{n, m}=V_{m, n}, \\
V_{n, m}^{\prime}=V_{m, n}^{\prime} .
\end{gathered}
$$

After Fourier transforming the interlayer free energy (1) and dropping the sixth-order terms, we find

$$
\begin{aligned}
F= & \frac{1}{2} \sum_{k} J_{q}\left(\vec{\sigma}_{k} \cdot \vec{\sigma}_{-k}\right)+J_{q}^{\prime}\left(\vec{\sigma}_{k} \times \vec{\sigma}_{-k}\right) \\
& +\frac{1}{4} \sum_{k, k^{\prime}, k^{\prime \prime}}\left(V_{-k-k^{\prime \prime}}+V_{-k-k^{\prime}}^{\prime}\right)^{\xi^{\prime}} \\
& \times\left(\vec{\sigma}_{k} \cdot \vec{\sigma}_{k^{\prime}}\right)\left(\vec{\sigma}_{k^{\prime \prime}} \cdot \vec{\sigma}_{-k-k^{\prime}-k^{\prime \prime}}\right) .
\end{aligned}
$$

As a consequence of Fourier transforming, $\vec{\sigma}_{k}$ is now an average tilt vector. From Eq. (2), $J_{q}$ must be real after Fourier transforming, as must $V_{-k-k^{\prime \prime}}$ and $V_{-k-k^{\prime \prime}}$. The chiral interaction $J_{q}^{\prime}$, on the other hand, must be imaginary.

Since the interactions are expected to be short ranged, we expand the various coefficients in terms of the layer spacing [or, equivalently, in terms of $\exp (i q)$ ]. For the second-order interaction term, since the interactions are short ranged, we include terms up to next-nearest neighbors in the nonchiral interactions $[5,6]$

$$
\text { " } J_{s_{q}}=J_{0}+J_{1} \cos (q)+J_{2} \cos (2 q)
$$

and up to nearest neighbor in the chiral interactions

$$
J_{q}^{\prime}=i J_{1}^{\prime} \sin (q)
$$

where $J_{1}^{\prime}$ is now a real number. Finally, expanding the fourth-order intęraction terms up to nearest neighbors yields

$$
V_{-k-k^{\prime \prime}}=V_{0}+V_{1} \cos \left(k+k^{\prime \prime}\right)
$$

and

$$
V_{-k-k^{\prime}}=V_{0}^{\prime}+V_{1}^{\prime} \cos \left(k+k^{\prime}\right)
$$

In order that the free energy of the system remain bounded from below, we must have

$$
\begin{gathered}
V_{0}+V_{0}^{\prime}>0, \\
V_{0}+V_{0}^{\prime}+V_{1}>0,
\end{gathered}
$$

and

$$
V_{0}+V_{0}^{\prime}+V_{1}^{\prime}>0
$$

In practice, it seems probable that $V_{0}$ and $V_{0}^{\prime}$ will have approximately the same order of magnitude as they cannot be rigorously distinguished from one another. Interlayer couplings are expected to be significantly smaller than intralayer interactions, however, so we expect that $V_{0} \gg V_{1} \approx V_{1}^{\prime}$.

Substituting (4) - (7) into the interlayer free energy (3) gives

$$
\begin{aligned}
F= & \frac{1}{2} \sum_{k}\left[\left(J_{0}+J_{1} \cos (q)+J_{2} \cos (2 q)\right]\right. \\
& \times\left(\vec{\sigma}_{k} \cdot \vec{\sigma}_{-k}\right)+i J_{1}^{\prime} \sin (q)\left(\vec{\sigma}_{k} \times \vec{\sigma}_{-k}\right) \\
& +\frac{1}{4} \sum_{k, k^{\prime}, k^{\prime \prime}}\left[\widetilde{V}_{0}+V_{1} \cos \left(k+k^{\prime \prime}\right)+V_{1}^{\prime} \cos \left(k+k^{\prime}\right)\right] \\
& \times\left(\vec{\sigma}_{k} \cdot \vec{\sigma}_{k^{\prime}}\right)\left(\vec{\sigma}_{k^{\prime \prime}} \cdot \vec{\sigma}_{-k-k^{\prime}-k^{\prime \prime}}\right),
\end{aligned}
$$

where $\widetilde{V}_{0} \equiv V_{0}+V_{0}^{\prime}$. To bring out the essential features, we first define a few new coefficients. Looking at the secondorder term in the interlayer free energy, we immediately see that it can easily be simplified into

$$
F_{(2)}=\sum_{k} a_{k}\left|\vec{\sigma}_{k}\right|^{2}
$$

where

$$
a_{k} \equiv J_{0}+J_{1} \cos (k)+i J_{1}^{\prime}\left(\hat{\vec{s}}_{k} \times \hat{\vec{s}}_{-k}\right) \sin (k)+J_{2} \cos (2 k)
$$

and the unit vector $\hat{\vec{s}}_{k_{k}}$ is defined as $\hat{\vec{s}}_{k}=\vec{\sigma} /|\vec{\sigma}|$. Next, we define the fourth-order coefficients:

$$
\begin{aligned}
b_{k} & =2\left[\tilde{V}_{0}+\cos (k)^{2} V_{1}+\dot{V}_{1}^{\prime}\right], \\
b_{k}^{\prime} & =\tilde{V}_{0}+V_{1}+\cos (2 k) V_{1}^{\prime}, \\
c_{k, k^{\prime}} & =\tilde{V}_{0}+V_{1} \cos \left(k+k^{\prime}\right)+V_{1}^{\prime},
\end{aligned}
$$




$$
\begin{gathered}
c_{k, k^{\prime}}^{\prime}=\widetilde{V}_{0}+V_{1}+V_{1}^{\prime} \cos \left(k+k^{\prime}\right), \\
c_{k_{m l}}=\widetilde{V}_{0}+\cos (2 k) V_{1}+\cos (2 k) V_{1}^{\prime} .
\end{gathered}
$$

This leaves us with a more manageable expression for the fourth-order terms in the interlayer free energy

$$
\begin{aligned}
& F_{(4)}=\sum_{k}\left(b_{k}\left|\vec{\sigma}_{k}\right|^{4}+b_{k}^{\prime}\left|\vec{\sigma}_{k} \cdot \vec{\sigma}_{k}\right|^{2}\right) \\
& +\sum_{k} \sum_{\left|k^{\prime}\right| \neq|k|}\left(c_{k, k^{\prime}}\left|\vec{\sigma}_{k}\right|^{2}\left|\vec{\sigma}_{k^{\prime}}\right|^{2}+c_{k, k^{\prime}}^{\prime}\left|\vec{\sigma}_{k} \cdot \vec{\sigma}_{k^{\prime}}\right|^{2}\right) \\
& +\sum_{\left|k_{m l 1}\right| \leqslant\left|k_{m l 2}\right|} c_{k_{m l}}\left(\vec{\sigma}_{k_{m l 1}} \cdot \vec{\sigma}_{k_{m l 1}}\right)\left(\vec{\sigma}_{k_{m l 1}} \cdot \vec{\sigma}_{k_{m l 2}}\right)
\end{aligned}
$$

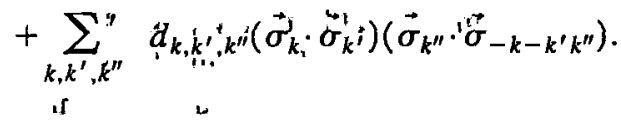

$$
\begin{aligned}
& \text { in it it }
\end{aligned}
$$

The final primied sưfimation contains only those combinations of $k, k^{\prime}$, and $k^{\prime \prime}$ 'that mix three distinct Fourier modes (i.e., those terms not included elsewhere in $\left.F_{(4)}\right)$. The sum over $k_{m l 1}$ and $k_{m l 2}$, on the other hand, contains only those pairs of wavevectors that satisfy the mode lock conditions

$$
\begin{gathered}
3 k_{m l 1}-k_{m l 2}=0, \\
3 k_{m l 1}+k_{m l 2}=2 \pi n ; \quad n= \pm 1, \pm 2, \ldots
\end{gathered}
$$

The first condition (20) reflects the origin of the mode lock terms as the $\left(\vec{\sigma}_{k} \cdot \vec{\sigma}_{k}\right)\left(\vec{\sigma}_{k} \cdot \vec{\sigma}_{-k^{\prime}}\right)$ term of the free energy (3). The second mode lock condition (21) is analogous to the condition for conserving momentum in an Umklapp process in a crystal. In an Umklapp process, electrons are able to "hop" across from one zone boundary to another while still conserving momentum to within a reciprocal lattice vector. Similarly, in heliclinic phases we are dealing with rotations in a plane, which are only specified up to a factor of $2 \pi$. Thus, a combination of rotations which advances the phase by $2 \pi$ is allowed the same as if the wave vectors had summed to zero. Since we are only keeping terms up to fourth order in the interlayer free energy, there are only two pairs of modes that satisfy the mode lock conditions (20) and (21). Those pairs are $(\pi / 3, \pi)$, and $(2 \pi / 3,2 \pi)$. Again, since rotations in a plane are always modulo $2 \pi$, the second pair is equivalent to $(2 \pi / 3,0)$. The pair $(\pi / 2, \pi / 2)$ does not satisfy the first mode lock condition (20), but could also possess interesting physics.

Substituting these new coefficients and adding together Eqs. (12) and (19) gives the final form for the interlayer free energy
TABLE I, Equivalence between (phenomenological) Landad coefficients and (interlayer) Landau-Ginzburg coefficients. Values of $k$ given are those appearing in the phenomenological Landau theory.

\begin{tabular}{cc}
\hline Landau & Landau-Ginzburg \\
\hline$a_{1}, a_{3}$ & $a_{k} \quad k=0,2 \pi / 3$ \\
$b_{1}, b_{3}$ & $b_{k} \quad k=0,2 \pi / 3$ \\
$b_{3}^{\prime \prime}$ & $b_{k}^{\prime} \quad k=2 \pi / 3$ \\
$c_{13}$ & $c_{k, k^{\prime}}^{\prime} \quad k, k^{\prime}=0,2 \pi / 3$ \\
$c_{13}^{\prime \prime}$ & $c_{k, k^{\prime}} \quad k, k^{\prime}=0,2 \pi / 3$ \\
$c_{r+1333}$ & $c_{k_{m l}} \quad k_{m l}=(0,2 \pi / 3)$ \\
\hline
\end{tabular}

$$
\begin{aligned}
F= & \sum_{k} a_{k}\left|\vec{\sigma}_{k}^{\prime}\right|^{2}+\sum_{k}\left(b_{k}\left|\vec{\sigma}_{k}\right|^{4}+b_{k}^{\prime}\left|\vec{\sigma}_{k} \cdot \vec{\sigma}_{k}\right|^{2}\right) \\
& +\sum_{k} \sum_{\left|k^{\prime}\right| \neq|k|}\left(c_{k, k^{\prime}}\left|\vec{\sigma}_{k}\right|^{2}\left|\vec{\sigma}_{k^{\prime}}\right|^{2}+c_{k, k^{\prime}}^{\prime}\left|\vec{\sigma}_{k} \cdot \vec{\sigma}_{k^{\prime}}\right|^{2}\right) \\
& +\sum_{k_{m l}} c_{k_{m l}}\left(\vec{\sigma}_{k} \cdot \vec{\sigma}_{k}\right)\left(\vec{\sigma}_{k} \cdot \vec{\sigma}_{-3 k}\right) \\
& +\sum_{k, k^{\prime}, k^{\prime \prime}}^{\prime} d_{k, k^{\prime}, k^{\prime \prime}}\left(\vec{\sigma}_{k} \cdot \vec{\sigma}_{k^{\prime}}\right)\left(\vec{\sigma}_{k^{\prime \prime}} \cdot \vec{\sigma}_{-k-k^{\prime} k^{\prime \prime}}\right) .
\end{aligned}
$$

\section{COMPARISON WITH LANDAU FREE ENERGY}

Compare Eq. (22) with our previous formulation of the Landau free energy in [2]

$$
\begin{gathered}
F=F_{0}+F_{i n t}, \\
F_{0}=a_{1} X^{2}+a_{3} Z^{2}+b_{1} X^{4}+b_{3} Z^{4}+c_{13}^{\prime} X^{2} Z^{2}, \\
F_{i n t}=-b_{3}^{\prime \prime} Z^{4} \sin ^{2}(2 \alpha) \cos ^{2}(\gamma)+c_{13} \cos ^{2}(\alpha) X^{2} Z^{2} \\
-c_{1333} X Z^{3} \cos (\alpha) \sqrt{1-\sin ^{2}(2 \alpha) \cos ^{2}(\gamma),}
\end{gathered}
$$

where $X$ is the magnitude of the ferroclinic order parameter, $Z$ is the magnitude of the $2 \pi / 3$ heliclinic order parameter, and $\alpha$ and $\gamma$ are angles related to the $2 \pi / 3$ heliclinic order parameter. Examining the two free energies term-by-term, one sees that the Landau coefficients $a_{q}, b_{q}^{\prime}$, and $c_{q, q^{\prime}}^{\prime}$ are given above by Eqs. (13), (14), and (16), respectively. Further, the Landau coefficients $b_{q}^{\prime \prime}$ and $c_{1333}$ in [2] are given above by Eqs. (15) and (18). Both of the Landau coefficients $c_{q, q^{\prime}}^{\prime \prime}$ and $c_{q, q^{\prime}}^{\prime \prime \prime}$ are given above by Eq. (17): The $d$ coefficients have no counterparts in the Landau free energy. These terms though, since they couple three different'wave, vectors are not likely to play a significant role in determining the phase diagram of the system. These results are summarized in Table I.

\section{PHYSICS BEYOND THE LANDAU THEORY}

Now that we have more physical expressions (13)-(18) for the various Landau coefficients in [2], we are able to make several additional comments about their relative mag- 
$+$

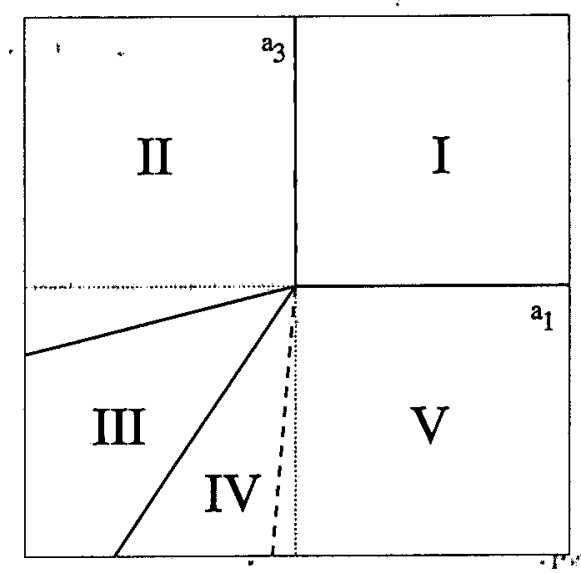

FIG. 2. Phase diagram as calculated previously. Region I and II are the the isotropic $(\mathrm{Sm}-A)$ and ferroclinic' ( $\mathrm{Sm}-C)$ phases, respectively. Region III is a chiral heliclinic phase, region IV is a planar heliclinic phase (similar to the $\mathrm{Sm}-C_{\dot{\gamma}}$ phase), zând region $\mathrm{V}$ is a second chiral heliclinc phase. Dotted lines" are the coordinate axes, solid lines are continuous transitions, heavy dashed lines are discontinuous transitions.

s.t.

nitudes and the relationships between them. Looking at the second-order coefficient $a_{k}$ one immediately'sees a possible competition between nearest neighbor and next-nearest neighbor if $J_{11}$ and $J_{2}$ have different signs. 'It is this same competition that drives the phase transitions in the discreet phenomenological models of Rovšek, Cepič, and Žekš $[5,6]$.

$J_{1}^{\prime}$, though, also plays an important role in determining the layer-by-layer structure of the system. As an odd function of $q$, the $J_{1}^{\prime}$ term causes the coefficient $a_{q_{r}}$ to distinguish between modes with helicity $q$ and modes with helicity $-q$. This is significant because all the other interactions in the interlayer free energy are even functions of $q$. While one would naturally expect on physical grounds that the chiral term is responsible for breaking the degeneracy between $q$ and $-q$, this was not readily apparent in our previous Landau free energy [2]. There, symmetry breaking between $q$ and $-q$ had to be introduced by fiat.

Next, looking at the definitions (16)-(18) one sees that the Landau coefficients $c_{13}, c_{13}^{\prime}$, and $c_{1333}$ are all positive numbers. While this is of only minor importance in determining the phase diagram, this too is a result that cannot be obtained from the form of the Landau free energy alone.

Finally, looking at $b_{k}^{\prime}$ in Eq. (15) one sees, given the bounds on the various terms in Eqs. (8) and (9), that $b_{k}^{\prime}$ is always positive. Consequently, the parameter $\cos (\gamma)$ of Eq. (23) is permitted to take on the values of zero and one [if $b_{k}^{\prime}$ were negative, $\cos (\gamma)$ would be identically zero]. In our previous paper [2] we considered two sequences of phase transitions since we were unable to determine the sign of $b_{k}^{\prime}$. Starting from the interlayer free energy, we see that the second, richer, sequence of phase transitions (reproduced in Fig. 2 ) is the correct sequence. This again is a result that cannot be derived from the Landau free energy formulation alone.

\section{CHIRALITY}

Spontaneous polarization, as observed in all known ferrielectric phases, can only occur in systems that lack a center of inversion. In liquid crystals, this means that the molecules are chiral. In a chiral system, as mentioned previously, the orientation of the liquid erystal rotates slowly throughout space. The formulation we have used for the interlayer free energy, however, does not yet account for this. In this section we will outline the effects that chirality has on our model.

At a microscopic level, chirality can be viewed as a layerby-layer rotation of the coordinate system. To correctly account for this rotation, though, it is easier to work with the complex field $\psi$ instead of the usual Cartesian vectors $\vec{\sigma}$ where

$$
\psi_{l} \equiv \vec{\sigma}_{l} \cdot \hat{\vec{x}}+i \vec{\sigma}_{l} \cdot \hat{\vec{y}}
$$

Rotations of $\vec{\sigma}$ by an angle $Q$ between adjacent layers simply changes the complex phase of $\dot{\psi}$,

$$
\psi_{l+1}=e^{i Q_{1}} \psi_{l}
$$

which is the chief virtue of this representation. Writing the nonchiral free energy (1) in terms of $\psi$ and Fourier transforming, we now find

$$
\begin{aligned}
F= & \frac{1}{2} \sum_{k}\left[J_{k}^{\prime}\left(\psi_{k} \psi_{k}^{*}\right)-i J_{k}^{\prime}\left(\psi_{k} \psi_{k}^{*}\right)\right] \\
& +\frac{1}{4} \sum_{k, k^{\prime}, k^{\prime \prime}}\left(V_{-k-k^{\prime \prime}}+V_{-k-k^{\prime}}\right) \\
& \times \psi_{k} \psi_{-k^{\prime}}^{*} \psi_{k^{\prime \prime}} \psi_{k+k^{r}+k^{\prime \prime}}^{*}
\end{aligned}
$$

In the above equation (24) and for the rest of this paper, we will use the convention that $\psi_{k}^{*}$ is the complex conjugate of the Fourier transform [i.e., $\left.\psi_{k}^{*}=\left(\sum_{l} \psi_{l} e^{i k l}\right)^{*}\right]$,

If the chiral interactions (via $J^{\prime}$ ) are small, then the chiral solutions should be equal to the nonchiral solutions plus some small chiral perturbation. Specifically, by calculating the $\psi_{k}$ for the nonchiral case and then adding some constant $Q \equiv 2 \pi / P$ to all the wave vectors in Eq. (24) we should be able to find the new chiral solutions using perturbation theory (to first order).

Upon doing so, however, it is apparent that the fourthorder term has only a weak dependence on the wave vector $Q$ since we expect intralayer interactions $\left(\widetilde{V}_{0}\right)$ to be significantly stronger than the layer-layer couplings $\left(V_{1}\right.$ and $\left.V_{1}^{\prime}\right)$ in the fourth-order terms. Therefore, this dependence is small and can safely be ignored. The remaining $Q$-dependent parts of the free energy are

$$
F_{Q}=\frac{1}{2} \sum_{k}\left(J_{k+Q}-i J_{k+Q}^{\prime}\right)\left|\psi_{k+Q}\right|^{2},
$$

where $\psi_{k}$ is obtained by minimizing the free energy of the nonchiral system. This portion of the free energy is easily minimized with respect to $Q$, from which we find

$$
\sum_{k}\left(\frac{\partial J_{k+Q}}{\partial Q}-i \frac{\partial J_{k+Q}^{\prime}}{\partial Q}\right)\left|\psi_{k+Q}\right|^{2}=0
$$

As we demonstrated in our initial paper [2], some of the heliclinic phases are confined to the $X Z$ plane and thus are 
achiral : Others, though, trace a helix along the $z$ axis and are thus manifestly chiral (e.g., they are asymmetric under $z \rightarrow$ $-z$ ). If the system is in a achiral phase then $\partial J_{k+Q} / \partial Q$ is an odd function of $Q$ and. so we must have $\partial J_{k+Q} / \partial Q$ $\approx Q\left(\partial^{2} J_{k+Q} /\left.\partial Q^{2}\right|_{Q=0}\right)$. Similarly, since it is an even function of $Q, \partial J_{k+Q}^{\prime} / \partial Q \approx$ const. These two expressions can be substituted back into Eq. (26) and the resulting equation solved for $Q$ which yields

$$
\left.Q \approx \frac{i \sum_{k}\left|\psi_{k+Q}\right|^{2} \partial J_{k+Q}^{\prime} / \partial Q}{\sum_{k}\left|\psi_{k+Q}\right|^{2} \partial^{2} J_{k+Q} / \partial Q^{2}}\right|_{Q=0} .
$$

This' implies that the heliclinic state should have a pitch of the same order of magnitude as that of the ferroclinic state. As the second derivative of $J$ cannot be predicted from the properties of the ferroclinic phase, however, no quantitative predictions for the pitch can be made.

If, on the other hand, the system is in a chiral phase (specifically, phases III and V in Fig. 2), the situation becomes more complicated. Since $\psi_{k}$ is not equivalent to $\psi_{-k}$ for nonzero $k$, there is no reason to assume that $\Sigma \partial J / \partial Q$ and $\Sigma \partial J^{\prime} / \partial Q$ are the same at $k$ and $-k$. Therefore, there is no reason to assume that $Q$ goes to zero as $J^{\prime}$ goes to zero. This, of course, stands to reason since as a chiral heliclinic phase, the system has a spontaneous "pitch" of its own. Therefore, we expect that this phase will have a chiral pitch rather larger than the pitch of the ferroclinic phase and that the sign of this pitch will be chosen by the sign of the chiral $J^{\prime}$ term.

\section{SUMMARY}

In conclusion, we have shown that it is possible to construct the Landau free energy used in our initial paper [2] starting from short-ranged interlayer interactions. Using a Landau-Ginzburg free energy, however, we are able to relate the various Landau coefficients to physical properties of the liquid crystal instead of leaving them as simple phenomenological parameters. This also allows us to make much better estimates of the sign and of the relative magnitude of the various coefficients than was possible from just the Landau free energy. Finally, we extended our model to include the effects of the natural chiral pitch. Our analysis of the intrinsic chirality is unsophisticated, but it nevertheless permits us to make rough estimates for the pitch in the achiral heliclinic phases and to make some qualitative statements about the effects of chirality in the chiral heliclinic phases.

\section{ACKNOWLEDGMENTS}

This research was supported by Grant No. DMR-8920147 from the National Science Foundation's Science and Technology Center for Advanced Liquid Crystalline Optical Materials (ALCOM).
[1] P. G. de Gennes and J. Prost, The Physics of Liquid Crystals, 2nd ed. (Oxford University Press, New York, 1993).

[2] J. Stott and R. Petschek, Phys. Rev. E 60, 1799 (1999).

[3] P. Mach et al., Phys. Rev. Lett. 81, 1015 (1998).
[4] R. Bruinsma and J. Prost, J. Phys. II 4, 1209 (1994).

[5] B. Rovšek, M. Čepič, and B. Žekš, Phys. Rev. E 54, 3113 (1997).

[6] M. Čepič and B. Žekš, Liq. Cryst. 20, 29 (1996). 